\title{
HAY FEVER AND HYPERTENSION IN THE US ADULT POPULATION $C \mathrm{Li}$, CL Cheung, TT Cheung, NR Samaranayake, BMY Cheung
}

\author{
Department of Medicine, The University of Hong Kong, Queen Mary Hospital, Hong Kong
}

OBJECTIVE: Hypertension is associated with inflammation. Whether the inflammation caused by allergic diseases such as allergic rhinitis can predispose to hypertension is controversial. Therefore, we studied the association between hay fever and hypertension in the United States National Health and Nutrition Examination Survey (NHANES).

METHODS: We analysed data on 1883 men and 2029 women in NHANES 2005-2006. We included participants aged 20 years or older who had valid data on hay fever and hypertension.

RESULTS: Of the participants, $13.5 \%$ had a previous diagnosis of hay fever and $36.2 \%$ had hypertension. There were ethnic differences in the prevalence of previous hay fever diagnosis $(\mathrm{P}<0.001)$ and hypertension $(\mathrm{P}<0.001)$. There was no significant association between previous hay fever diagnosis and hypertension in men in any age-group. The association between previous hay fever diagnosis and hypertension in women was significant in those aged 20 to 39 years (odds ratio $[\mathrm{OR}]=2.59,95 \%$ confidence interval $[\mathrm{CI}]: 1.26-5.30, \mathrm{P}=0.013)$. The association between previous hay fever diagnosis and hypertension in women aged form 20 to 39 years remained significant after adjustment for age, race, and body mass index (OR=2.74, 95\% CI: 1.48-5.06, $\mathrm{P}=0.003$ ). After further adjustment for physical activity, alcohol consumption and smoking, the association was not attenuated $(\mathrm{OR}=2.68,95 \% \mathrm{CI}$ : $1.38-5.18, \mathrm{P}=0.006)$. Further adjustment for liver enzymes, $\mathrm{C}$-reactive protein and immunoglobulin $\mathrm{E}$ level attenuated the association slightly $(\mathrm{OR}=2.72,95 \% \mathrm{CI}: 1.19-6.22, \mathrm{P}=0.021)$.

CONCLUSIONS: In this nationally representative population-based survey, previous hay fever diagnosis is not significantly associated with hypertension in adults except for young women aged 20 to 39 years. Further work is needed to confirm that this is a true association. 